\title{
ESTADO ACTUAL DE LAS POBLACIONES DE TORTUGAS TERRESTRES GIGANTES (Geochelone spp., Chelonia: Testudinae) EN LAS ISLAS GALÁPAGOS
}

\section{POPULATION STATUS OF GIANT LAND TORTOISES (Geochelone spp., Chelonya: Testudinae) FROM THE GALAPAGOS ISLANDS}

\author{
Cruz Márquez ${ }^{1}$, David Wiedenfeld ${ }^{2}$, Howard Snell ${ }^{3}$, Thomas Fritts ${ }^{4}$, Craig MacFarland ${ }^{5}$, Washington Tapia ${ }^{6}$ y \\ Sixto Naranjo ${ }^{7}$
}

\begin{abstract}
Resumen
Se estudió el estado actual de las poblaciones de tortugas terrestres gigantes (Geochelone) en las islas Galápagos. Métodos de distribución geográfica, marca-recaptura, estructura, densidad, relación de sexo, depredación y amenazas, son usados en el contexto del estudio. Resultados de registros antiguos indican que la distribución y demografía poblacional actual de las tortugas gigantes está muy por debajo de la que existió en los siglos XVI-XVIII y fue diferente entre las de 1974 y 1992-2002. La distribución poblacional actual es similar a la de 1974 estimada por MacFarland. Demografías poblacionales actuales son diferentes a las de 1974. Los valores de $t$ estuvieron entre 29 á 175, P <0.001. Las densidades poblacionales variaron desde 57 á 799 tortuga $/ \mathrm{km}^{2}$. Las estructuras poblacionales actuales de $G$. vandenburghi y G. nigrita, en los machos, hembras y juveniles son diferentes con las de 1974. Los machos, las hembras y los juveniles, los tamaños poblacionales actuales de G. ephippium y microphyes, no son diferentes a las de 1974. Las estructuras poblacionales de las seis poblaciones remanentes, las actuales, con las de 1974, en algunos de los tres grupos de sexos son diferentes, y en otros no (Ji-Cuadrados lo muestran). Las proporciones de sexo (hembras: machos) actuales de las tortugas, en mayoría son superiores a las de 1974. En las islas Galápagos, en la actualidad cada vez son menos las poblaciones de tortugas gigantes que mantienen depredadores y competidores en su ambiente; ya que, las amenazas potenciales de las poblaciones de tortugas supervivientes están siendo erradicadas cada día, pero aún se mantienen algunas. Los cerdos y perros cimarrones son erradicados de Santiago e Isabela respectivamente; pero aún quedan los domésticos como amenazas potenciales permanentes. Las islas Pinta Española y Santa Fe, actualmente no tienen cabras, pero éstas habitan en Isabela, Santiago, Santa Cruz y San Cristóbal.
\end{abstract}

Palabras Claves: Estado poblacional de las Tortugas Gigantes, Islas Galápagos.

\begin{abstract}
The present status of populations of giant land tortoises (Geochelone spp) in the Galápagos Islands was studied. Geographical distribution, mark and recapture methods, population structure, population density, sex ratios, predation and other threats were considered in this context. Old records show that the present distribution and population demography of giant tortoises is much more restricted than that seen during the $16^{\text {th }}$ to $18^{\text {th }}$ centuries and, although there were further population changes between 1974 and 1992 - 2002, the population distribution is still similar to that estimated by McFarland (1974). Present population demographics differ from those of 1974, the values of Student's t range from 29 to $175(\mathrm{P}<0.001)$. Population densities vary from 57 to 799 tortoises $/ \mathrm{km}^{2}$. The present population structures of $G$. vandenburghi and nigrita for males, females and juveniles differ from those of 1974. However, these three population classes in $G$. ephippium and $G$. microphyes have remained unchanged since 1974. Comparing the present population structures of the six remaining populations with those of 1974, Chi-squared tests show that population structure differs in some populations but not others. Present sex ratios (females: males) of tortoises are in general higher than those of 1974. There are now fewer populations of tortoises affected by predators and competitors. Potential threats to surviving tortoise populations have been generally eliminated. Wild pigs and dogs in both Santiago and Isabela islands have been eradicated although domestic ones remain as a permanent, potential threat. The islands of Pinta, Espanola and Santa Fe no longer have goats, although they are still present in Isabela, Santiago and San Cristobal.
\end{abstract}

Key words: Populations status, giant tortoises, Galapagos Islands. 


\section{Introducción}

Las tortugas terrestres gigantes, fueron abundantes en todos los continentes, pero actualmente solo habitan en el atolón de Aldabra en las islas Seychelles (Geochelone gigantea) y en las islas Galápagos (Gunther, 1875; MacFarland et al., 1974). Las poblaciones de tortugas fueron abundantes en las islas Galápagos en los siglos XVI hasta el XVIII, (Porter, 1815; Vandenburgh, 1914). Distribuidas en las islas donde ahora habitan (Figura 1), ellas fueron diezmadas por la presencia de filibusteros (piratas, balleneros, cazadores de focas, mercaderes y colonos. Townsend, 1925; Slevin, 1959). Los problemas de depredación que sufrieron estos reptiles por mamíferos cimarrones y los humanos en Isabela, Santiago, San Cristóbal, Floreana, Española, Pinta, Santa Fe, Santa Cruz y Pinzón, fueron documentados desde hace muchas décadas (Beck, 1903).

Estudios taxonómicos mencionan que en las islas Galápagos se presentan poblaciones del género Geochelone (Fitzinger, 1835) con 14 especies de tortugas terrestres gigantes, distribuidas en 10 islas (Tabla 1, Gunther, 1875, 1877; Baur, 1889; Van
Denburgh, 1914). Muchas de las poblaciones de tortugas fueron disminuidas por la depredación de huevos, juveniles y adultos por mamíferos cimarrones y el hombre. En el 1964 ya se comprueba la extinción de 3 especies en Santa Fe y Floreana por captura humana y una de dudosa existencia en Rábida. En los años 1964-1974 (periodo de la última revisión entera de las poblaciones de las tortugas; MacFarland et al. , 1974) sobrevivían entonces 12 especies, 10 con poblaciones pequeñas recuperables y dos no recuperables (Figura 1). Estas dos fueron la de Pinta (G. abingdoni) porque se la considera extinta en la isla, solamente queda un ejemplar macho "Solitario Jorge" que se lo mantiene en cautiverio desde 1972 en la Estación Científica Charles Darwin (FCD) y el Parque Nacional Galápagos (PNG) y la especie de Fernandina ( $G$. phantastica) que se extinguió con la erupción de 1977, cuando el magma cubrió la mayor parte de la isla.

La taxonomía de las tortugas gigantes de Galápagos hasta el momento es muy discutida por varios científicos. Algunos las consideran subespecies $\mathrm{y}$ otros especies, y en el pasado le asignaron algunos

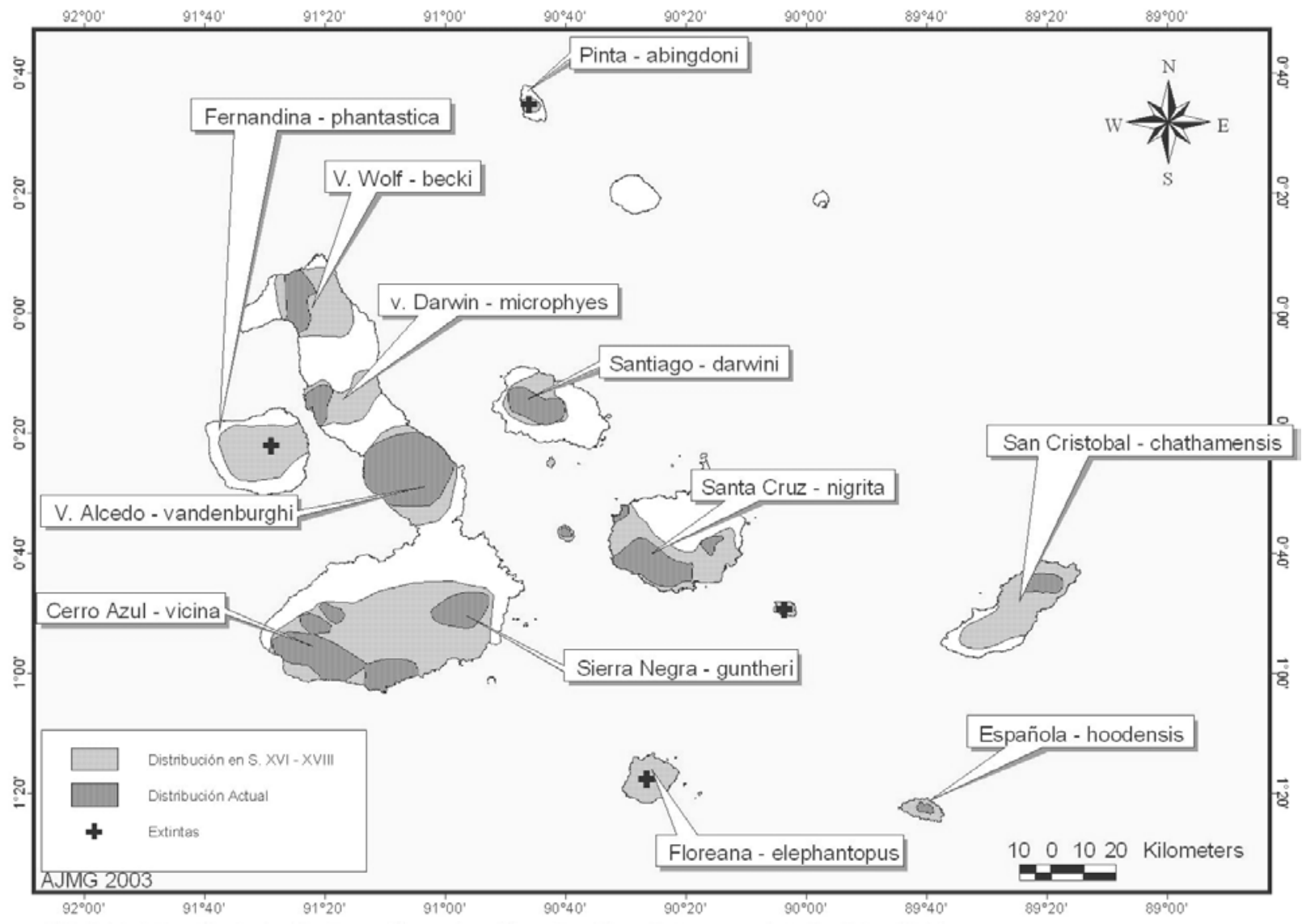

Figura 1. Distribución de las tortugas terrestres gigantes (Geochelone nigra) de las islas Galápagos en los siglos XVI - XVIII (VanDenburgh, 1914), 1964 - 1974 y la actual 1992 - 2002. Se indica también las 4 poblaciones de tortugas gigantes que se han extinguido hasta la fecha. 
Tabla 1. Especies de tortugas gigantes, morfología del carapacho y tipo de ambiente.

$=$ islas de ambiente árido

** = isla de ambiente semiárido

$* * *$ = isla de ambiente húmedo.

\begin{tabular}{|c|c|c|}
\hline Isla o volcán & $\begin{array}{l}\text { Especie de } \\
\text { tortuga }\end{array}$ & $\begin{array}{l}\text { Morfotipo de } \\
\text { carapacho }\end{array}$ \\
\hline Española* & G. hoodensis & Montura \\
\hline Pinzón* & G. ephippium & Montura \\
\hline Pinta* & G. abingdoni & Montura \\
\hline San Cristóbal** & G. chathamensis & Intermedio \\
\hline Santiago*** & G. darwini & Cúpula \\
\hline Santa Cruz*** & G. nigrita & Cúpula y Montura \\
\hline $\begin{array}{l}\text { Isabela volcán Cerro } \\
\text { Azul*** }\end{array}$ & G. vicina & Cúpula \\
\hline $\begin{array}{l}\text { Isabela volcán Sierra } \\
\text { Negra*** }\end{array}$ & G. guntheri & Intermedia y Cúpula \\
\hline $\begin{array}{l}\text { Isabela volcán } \\
\text { Alcedo*** }\end{array}$ & $\begin{array}{l}\text { G. } \\
\text { vandenburghi }\end{array}$ & Cúpula \\
\hline $\begin{array}{l}\text { Isabela volcán } \\
\text { Darwin** }\end{array}$ & G. microphyes & Intermedia y Cúpula \\
\hline Isabela volcán Wolf** & G. becki & $\begin{array}{l}\text { Montura, Intermedia } \\
\text { y Cúpula }\end{array}$ \\
\hline Fernandina & G. phantastica & Montura \\
\hline Santa Fe & G. spp. & Montura \\
\hline Floreana & $\begin{array}{l}\text { G. } \\
\text { galapagoensis }\end{array}$ & Montura \\
\hline
\end{tabular}

nombres científicos. En 1835, Darwin dio el nombre de Testudo nigra, Gunther $(1875,1877)$ asignó a todas las especies el nombre genérico de Testudo, con las respectivas especies actuales, y solamente llamó $T$. nigra a la especie de la isla Santiago (Baur, 1889). Harland $(1827,1835)$ usó por primera vez el nombre de Geochelone elephantopus, el cual fue utilizado hasta 1988. Ernst \& Barbour (1989) y Fritts (2001) consideraron a todas las subespecies como especies únicas individuales. Prichard (1996) sugirió volver a reconsiderar Geochelone nigra, para todas las subespecies de tortugas gigantes de Galápagos. Caccone et al. $(1999,2002)$ y Ciofi et al. (2002), en estudios genéticos de ADN satelital, volvieron a asignar el nombre específico de Geochelone nigra, manteniendo los mismos nombres subespecíficos, ya utilizados por Darwin en 1835 y Van Denburgh (1914). Para este trabajo, se usará el género Geochelone y los nombres de las especies correspondientes en cada una de las islas y/o volcanes, según la nomenclatura de Ernst \& Barbour (1989) y Fritts (2001).
Hace 30 años se realizó un estudio similar por MacFarland et al. (1974), desde entonces no se ha elaborado ningún informe que presente una evaluación del estado actual de las poblaciones de tortugas terrestres gigantes en Galápagos. Por eso este documento pretende mostrar el estado actual (en el período 1992 - 2002), la distribución, demografía, proporción de sexo, estructura actual poblacional, depredación y amenazas de las poblaciones de tortugas del Archipiélago. También se pretende comparar el estado actual de las tortugas con las estimadas en 1974 y evaluar los 40 años de labor conservacionista desplegados por el PNG y la FCD, protegiendo y conservando las poblaciones de tortugas gigantes, que estuvieron más amenazadas por el hombre y organismos cimarrones introducidos.

\section{Materiales y Métodos Área de estudio}

Se estudiaron las tortugas en distintos escenarios, en ambientes áridos, semiáridos y húmedos: Ambiente árido: Española $\left(-1^{\circ}-38^{\prime}-\mathrm{S},-89^{\circ}-67^{\prime}-\mathrm{O}\right)$, con una superficie de $60.5 \mathrm{~km}^{2}$ y altitud máxima $206 \mathrm{msnm}$, con una precipitación anual de $10 \mathrm{~mm}$ en años secos y $600 \mathrm{~mm}$ en años lluviosos, con una temperatura promedio anual de $23.8^{\circ} \mathrm{C}$. La cobertura vegetal más común está compuesta por lianas, hierbas, arbustos y árboles. Incluye especies de aves: como pinzones de cactus (Geospiza conirostris), cucuve (Nesomimus macdonaldi), paloma silvestre (Zenaida galapagoensis), gavilán de Galápagos (Buteo galapagoensis) y canario maría (Dendroica petechia). Las tortugas comparten la isla con dos otros reptiles, las culebras (Philodryas hoodensis) y lagartijas (Microlophus delanoni).

Ambiente semi-árido: Pinzón $\left(-0^{\circ}-36^{\circ}-\mathrm{S},-90^{\circ}-\right.$ $40^{\prime}$ - O), con una superficie de $18.15 \mathrm{~km}^{2}$, una altitud máxima de 458 msnm, y San Cristóbal (-0 - 49' - S, $\left.89^{\circ}-25^{\prime}-\mathrm{O}\right)$, con una superficie de $558.09 \mathrm{~km}^{2}$, una altitud de $730 \mathrm{msnm}$. Ambas islas son de origen volcánico, con una precipitación anual de $368 \mathrm{~mm}$ en años secos y $860 \mathrm{~mm}$ en años lluviosos, una temperatura máxima promedio anual de $25.3^{\circ} \mathrm{C}$. La cobertura vegetal más común esta compuesta de árboles, arbustos, lianas y hierbas. Mantienen algunas especies de aves como pinzones (Geospiza spp.), palomas silvestres, cucuves (Nesomimus melanotis en San Cristóbal), canario maría, papamoscas (Myiarchus magnirostris), cuclillo (Coccyzus melacoryphus) y gavilán en Pinzón; San Cristóbal no tiene gavilán. De reptiles: hay varias especies como culebras (Antillophis slevini) y lagartijas de lava (Microlophus duncanensis) en Pinzón, culebras (Alsophis biserialis), lagartijas de lava (Microlophus bivitatus), geckos (Phyllodactylus leei y $P$. darwini) en San Cristóbal.

Ambiente húmedo: Santa Cruz ( $-0^{\circ}$ - $37^{\prime}$ - S, - $90^{\circ}$ $\left.21^{\prime}-\mathrm{O}\right)$, con una superficie de $985.55 \mathrm{~km}^{2}$, una altitud 
máxima de 864 msnm, con una precipitación promedio anual de $640 \mathrm{~mm}$, y temperatura máxima promedio anual de $26.7^{\circ} \mathrm{C}$ : Santiago $\left(-0^{\circ}-16^{\prime}-\mathrm{S}\right.$, $\left.90^{\circ}-42^{\prime}-\mathrm{O}\right)$, con una superficie de $584.65 \mathrm{~km}^{2}$, con una altitud máxima de $907 \mathrm{msnm}$, con una precipitación promedio anual de $381 \mathrm{~mm}$, y una temperatura máxima promedio anual de $25.3^{\circ} \mathrm{C}$ : Isabela $\left(-0^{\circ}-25^{\prime}-\mathrm{S},-91^{\circ}-6^{\prime}-\mathrm{O}\right)$, en una superficie de $4588 \mathrm{~km}^{2}$, y una altitud máxima de 1028-1700 msnm, con una precipitación promedio anual de 436 $\mathrm{mm}$, y temperatura máxima promedio anual de $24.2^{\circ} \mathrm{C}$. Todas son de origen volcánico. La cobertura vegetal más común es de árboles, arbustos, hierbas, helechos y áreas de pampa. Mantienen algunas especies de aves, tal como pinzones (Geospiza spp.), palomas silvestres, cucuves (Nesomimus parvulus), cuclillos, canario maría, papamoscas, pachay (Lateralus spilonotus), gallareta (Neocrex erythrops), gallinula (Gallinula chloropus), gavilán, garzas (Herodyas spp.), golondrinas (Progne modesta), flamencos (Phoenicopterus ruber), patillos (Anas bahamensis) y pájaro brujo (Pyrocephalus rubinus); reptiles como culebras (Antillophis steindachneri) en Santa Cruz, (Alsophis biserialis) en Isabela y Santiago, lagartijas de lava (Microlophus albemarlensis) en Santa Cruz, Isabela y Santiago, iguanas terrestres (Conolophus subcristatus) en Santa Cruz e Isabela y geckos (Phyllodactylus galapagoensis) en Isabela, Santa Cruz y Santiago (Figura 1).

\section{Morfología de Carapachos}

Las especies de tortugas de las islas Galápagos, las conforman tres morfotipos dependiendo de las características climáticas y zonas vegetales de las islas (húmeda, árida y semiárida). Las tortugas con carapacho de forma Domo o Cúpula, por lo general habitan en las zonas sureñas de las islas grandes y húmedas. Es la tortuga de mayor tamaño y peso, un macho puede alcanzar $1.70 \mathrm{~m}$ de longitud de carapacho, un peso de $320 \mathrm{~kg}$; una hembra hasta los $120 \mathrm{~cm}$ en LC y $100 \mathrm{~kg}$ de peso, tienen extremidades y cuello corto, son lentas en el desplazamiento. La tortuga con carapacho en forma de silla de montar o de morfotipo montura viven en islas bajas y áridas. Son las tortugas más pequeñas del Archipiélago, es la verdadera tortuga Galápago. Los machos pueden alcanzar hasta los $100 \mathrm{~cm}$ en longitud de carapacho y un peso de $85 \mathrm{~kg}$ (depende de la isla). Las hembras alcanzan los $70 \mathrm{~cm}$ de LC y $40 \mathrm{~kg}$ de peso. Estas tortugas tienen cuello y extremidades largas, su locomoción, es muy rápida con relación a las anteriores. Las tortugas de morfología de carapacho intermedia habitan en islas semiáridas y son, de tamaño intermedio. Los machos alcanzan los $112 \mathrm{~cm}$ en longitud de carapacho y un peso de hasta $80 \mathrm{~kg}$, con cuello y extremidades intermedias (Tabla 1).

\section{Métodos usados en el campo}

El estudio fue realizado con la captura y marcación - recaptura de tortugas en las varias poblaciones. En la década del 1964 - 1974, como en la década del 1992 2002, el estudio se realizó en distintas fechas y años. Durante 1992-2002, el número de observadores varió en 3,6 , y 9, dependiendo de la disponibilidad de personas y del tamaño de la población donde se marcaron y recapturaron las tortugas. En las poblaciones pequeñas como la de Pinzón, y los volcanes de Isabela (a excepción de volcán Alcedo) fueron con tres observadores. En las poblaciones medianas de tortugas como Santiago, San Cristóbal, Santa Cruz y Española, fueron dos grupos de tres personas cada uno. Las poblaciones grandes como la de Alcedo, incluyeron tres y cuatro observadores en cada grupo (Tabla 2 ).

Tabla 2. Métodos y variables usadas por sitio de estudio. $\mathrm{LC}=$ Largo curvo del carapacho, $\mathrm{v}=$ volcán.

\begin{tabular}{|l|l|l|l|}
\hline Parámetros & \multicolumn{1}{|c|}{ Métodos } & \multicolumn{1}{|c|}{ Subtítulos } & \multicolumn{1}{|c|}{$\begin{array}{c}\text { Sitios de } \\
\text { trabajo }\end{array}$} \\
\hline Distribución & $\begin{array}{l}\text { Se usó GPS con } \\
\text { Track } \\
\text { programado }\end{array}$ & $\begin{array}{l}\text { Programa } \\
\text { G7Towin }\end{array}$ & $\begin{array}{l}\text { En todos los } \\
\text { lugares }\end{array}$ \\
\hline Demografía & $\begin{array}{l}\text { Petersen } \\
\text { Jolly-Seber }\end{array}$ & $\begin{array}{l}\text { Marca con } \\
\text { pintura } \\
\text { Recaptura }\end{array}$ & $\begin{array}{l}\text { Pinzón, } \\
\text { v.Darwin, etc } \\
\text { v. Alcedo, Sta. } \\
\text { Cruz, } \\
\text { v. Cerro Azul, } \\
\text { etc. }\end{array}$ \\
\hline Densidad & $\begin{array}{l}\text { Longitud de las } \\
\text { transectas 2-8 } \\
\text { Km }\end{array}$ & $\begin{array}{l}\text { Ancho de las } \\
\text { transectas } 50- \\
\text { 200m }\end{array}$ & $\begin{array}{l}\text { En todos los } \\
\text { lugares }\end{array}$ \\
\hline Estructura & $\begin{array}{l}\text { El tamaño en } \\
\text { LC. cm }\end{array}$ & $\begin{array}{l}\text { Tamaños de } \\
\text { clases de 10 en } \\
10 \text { en cm }\end{array}$ & $\begin{array}{l}\text { Para todas las } \\
\text { poblaciones- } \\
\text { lugares }\end{array}$ \\
\hline $\begin{array}{l}\text { Proporción } \\
\text { de Sexo }\end{array}$ & Machos/hembras & Hembras/machos & $\begin{array}{l}\text { En todos los } \\
\text { sitios }\end{array}$ \\
\hline $\begin{array}{l}\text { Mortalidad } \\
\text { Natural y } \\
\text { Depredación }\end{array}$ & $\begin{array}{l}\text { Cuantificación } \\
\text { de animales } \\
\text { muertos por } \\
\text { causa natural o } \\
\text { por depredación }\end{array}$ & $\begin{array}{l}\text { Huevos, crías o } \\
\text { adultos }\end{array}$ & $\begin{array}{l}\text { En todos los } \\
\text { sitios }\end{array}$ \\
\hline $\begin{array}{l}\text { Observación de } \\
\text { presencia de } \\
\text { depredadores }\end{array}$ & $\begin{array}{l}\text { Hormigas, } \\
\text { perros, cerdos, } \\
\text { gavilanes y el } \\
\text { hombre }\end{array}$ & $\begin{array}{l}\text { En todos lo } \\
\text { lugares de } \\
\text { muestreo }\end{array}$ \\
\hline
\end{tabular}

Desde 1992 al 2002, la distribución de las tortugas en cada una de las poblaciones fue mapeada vía GPS, y los datos fueron desplegados en mapas usando el programa mediante un Sistema de Información Geográfica (GIS, Figuras 1 y 2). MacFarland (1974) presentó una distribución de las tortugas usando altímetro y ubicó en mapas con gradientes altitudinales.

Las estimaciones poblacionales de las tortugas en cada uno de los lugares de muestreo, se realizó mediante el método de Marca-Recaptura de Petersen (para poblaciones pequeñas) y modelos modificados Jolly-Seber (para las poblaciones grandes; Crebs, 


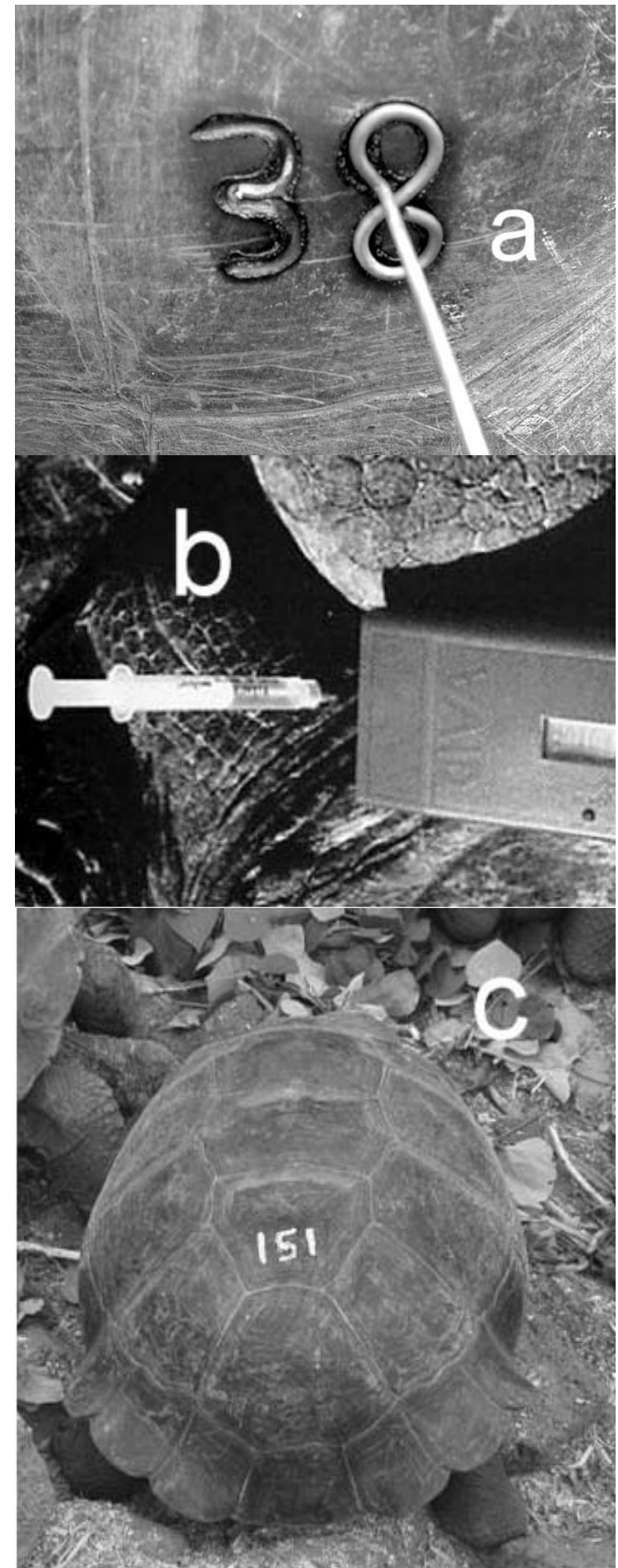

Figura 2 a-c. Tortugas que muestran la forma de los tres sistemas de marcación que han sido usados $(\mathrm{a}=$ marcación con herradura, $\mathrm{b}=$ marcación con Pits, trasmisor de implantación permanente y $\mathrm{c}=$ marcación con pintura, para una revisión rápida) para marcar a cada una de las tortugas que fueron capturadas en la marcación - recaptura, para las estimaciones poblacionales (Fotos: Cruz Márquez).
1989). Las tortugas fueron marcadas con pintura, herradura en la cuarta placa vertebral y etiquetas PITs (transponder de implantación permanente) implantado en forma subcutánea en la región femoral izquierda (Figura 3 a-c). MacFarland (1974) para mostrar las estimaciones poblacionales, usó el método de MarcaRecaptura de Lincoln.

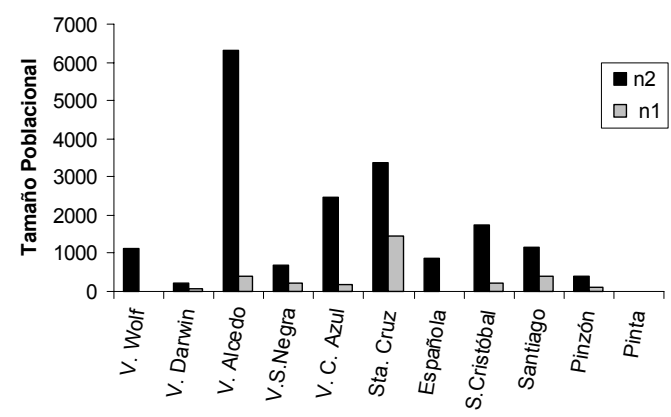

Figura 3. Estimaciones poblacionales de tortugas terrestres gigantes (Geochelone spp. ) actual (1992 $\left.2002, \mathrm{n}_{2}\right)$ y del pasado $\left(1974, \mathrm{n}_{1}\right)$ de seis islas del Archipiélago de las Galápagos.

Los datos de tamaños corporales en longitud de carapacho (LC) de las tortugas medidas se clasificaron en tamaños de clase de 10 en $10 \mathrm{~cm}$, desde el menor al mayor. Para la densidad poblacional, se midió la distancia a la tortuga más cercana y distante a la línea o transecta, tal como lo realizó MacFarland et al. (1964-1974).

Los transeptos fueron determinados en forma aleatoria en los distintos caminos usados por las tortugas, para su desplazamiento cotidiano en busca de alimento. Las medidas de los transeptos fueron variables. La longitud fue de $2-8 \mathrm{~km}$ y el ancho de acuerdo a la visibilidad que permitía el bosque entre 50 a 200 m (Tabla 3$)$.

Para cuantificar mortalidad y amenazas, en todas las poblaciones de tortugas, se hizo observación de presencia de individuos muertos por causa natural o depredación, que tipo de depredador y a que nivel (huevos crías o adultos). Estos fueron clasificados en seis grupos: 1) Las tortugas de muerte natural, su carapacho estuvo intacto sin desmembramiento del cuerpo. 2) Las tortugas sacrificadas por humanos, su cuerpo estuvo desmembrado con un hacha o machete. 3) Las jóvenes tortugas atacadas por animales como cerdos, su carapacho fue encontrado abierto por la parte dorsal en forma desordenada. 4) Los neonatos y juveniles de tortugas que fueron atacados por gavilanes, en su carapacho se observaba claramente las garras, sus huesos rotos y consumida su carne. 5) Tortugas por muerte natural, el cuerpo fue encontrado intacto. 6) Los nidos fueron removido por los cerdos hasta comerse los huevos y las cáscaras fueron encontradas en el substrato removido. Al excavar los nidos, verificar presencia de hormigas Solenopsis spp. 
en el nido atacando a los neonatos que habían iniciado el proceso de eclosión.

Tabla 3. Tamaños poblacionales estimados de las tortugas terrestres gigantes (Geochelone) de las islas Galápagos hasta 1973 por Macfarland et al. (1974). ( $\mathrm{N}=$ Tamaño estimado de población de las tortugas gigantes en un lapso de 9 años, desde 1965 - 1973). La mayoría de los intervalos mínimos estimados, son mayores a la $\mathrm{N}$ estimada, se exceptúan G. guntheri y G. ephippium, cuyos valores mínimos son menores a los $\mathrm{N}$ estimados. La cantidad de tortugas juveniles repatriadas hasta 1974 fue mínima, el programa comenzó en 1965 con la población de la isla Pinzón, para 1973, ya se habían repatriado 122 animales a Pinzón y 14 individuos liberados a volcán Wolf. La información de este Tabla, nos permitirá efectuar comparaciones con los estimados actuales de población.

\begin{tabular}{|c|c|c|}
\hline Isla o Volcán & Población & $\begin{array}{l}\text { Área } \\
\left(\mathrm{km}^{2}\right)\end{array}$ \\
\hline Isabela v. Wolf & G. becki & 5.0 \\
\hline " v. Darwin & G. microphyes & 4.1 \\
\hline “ $\quad$ v. Alcedo & G. vandenburghi & 7.91 \\
\hline “ $\quad$ v. Sierra Negra & G. guntheri & 5.69 \\
\hline “ v. Cerro Azul & G. vicina & 16.61 \\
\hline Santa Cruz & G. nigrita & 4.65 \\
\hline Española & G. hoodensis & 4.30 \\
\hline San Cristóbal & G. chathamensis & 3.08 \\
\hline Santiago & G. darwini & 9.40 \\
\hline Pinzón & G. ephippium & 4.0 \\
\hline Pinta & G. abingdoni & 0 \\
\hline
\end{tabular}

Los análisis estadísticos usados fueron: para la comparación de los tamaños poblacionales estimados entre 1964 - 1974 y 1992 - 2002, se transformaron los Errores Estándar a Desviación Estándar, y luego a varianzas, se usó la prueba $\mathrm{t}$ de Student, que puede comparar diferencias entre ambas temporadas. JiCuadrado, fue usada para comparar las estructuras poblacionales de machos hembras y juveniles, entre los grupos de tamaños de clases de 1974 y los actuales (Zar, 1999).

\section{Resultados}

\section{Distribución}

La distribución geográfica actual, es similar a la que presentó MacFarland (1974), pero diferente en lo referente a la historia del pasado de cada población (Siglos XVI-XVIII), solo difieren en nuevos lugares, pequeñas extensiones de áreas y los tamaños de muestras de individuos marcados, como en la estimación poblacional de cada isla o población (Figura 1).

\section{Demografía}

En comparación, en el estudio actual, los tamaños poblacionales más bajos lo mostraron, las poblaciones de Pinzón, volcán Sierra Negra, Volcán Darwin y la isla Española con 532, 694, 818 y 860 individuos respectivamente. Las más altas estuvieron entre Santa Cruz y los volcanes Cerro Azul y Alcedo, con 3391, 2574 y 6320 individuos respectivamente. Los tamaños intermedios, lo presentaron las islas y volcanes remanentes; mas 3261 repatriados (Tabla 5 y Figura 4). Todas las estimaciones poblacionales, realizadas entre 1992 hasta el 2002, fueron significativamente diferentes a las estimadas por MacFarland en 1974 (Tabla 5). Las comparaciones estadísticas mostraron diferencias en el tamaño de los estimados entre 1974 y $1992-2002$ (ts $=29-175$, g. $1 .=307-6318, \mathrm{P}<$ 0.0005), se exceptúan las poblaciones de tortugas gigantes de Santa Cruz y la de volcán Wolf, de los datos existentes, no se pudo obtener Errores Estándar, de las estimaciones anteriores, y Pinta que existe un solo individuo macho.

Tabla 4. Estimaciones poblacionales de las tortugas terrestres gigantes de las islas Galápagos entre 1992 al 2002. n = número de tortugas capturadas; $\mathrm{N}^{*}=$ población actual estimada; Intervalos = población mínima y máxima estimada; Densidad $/ \mathrm{km}^{2}=$ cantidad de tortugas que actualmente se estima que viven en un kilómetro cuadrado en el sitio de marcación; Fuente $=$ es el autor y año del monitoreo: $\mathrm{CM}=$ Cruz Márquez, PNG = Parque Nacional Galápagos, GM = Germán Morillo; Individuos repatriados = Tortugas jóvenes desarrolladas en cautiverio y devueltas a su lugar de origen, como incremento a la población.

\begin{tabular}{|c|c|c|c|c|}
\hline $\begin{array}{l}\text { Isla o } \\
\text { Volcán }\end{array}$ & Especie & $\mathrm{n}$ & Intervalo & 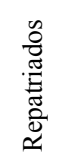 \\
\hline v. Wolf & G. becki & 0 & $1000-2000$ & 14 \\
\hline v. Darwin & G. microphyes & 65 & $500-1000$ & 0 \\
\hline v. Alcedo & $\begin{array}{l}\text { G. } \\
\text { vandenburghi }\end{array}$ & 402 & $3000-5000$ & 0 \\
\hline $\begin{array}{l}\text { v. Sierra } \\
\text { Negra }\end{array}$ & G. guntheri & 219 & $200-300$ & 0 \\
\hline $\begin{array}{l}\text { v. Cerro } \\
\text { Azul }\end{array}$ & G. vicina & 196 & $400-600$ & 0 \\
\hline Santa Cruz & G. nigrita & 1460 & $2000-3000$ & 0 \\
\hline Española & G. hoodensis & 15 & $20-\quad 30$ & 0 \\
\hline $\begin{array}{l}\text { San } \\
\text { Cristóbal }\end{array}$ & $\begin{array}{l}\text { G. } \\
\text { chathamensis }\end{array}$ & 213 & $500-700$ & 0 \\
\hline Santiago & G. Darwin & 389 & $500-700$ & 0 \\
\hline Pinzón & G. ephippium & 100 & $150-200$ & 122 \\
\hline Pinta & G. abingdoni & 1 & Muy pequeña & 0 \\
\hline Total & & 3060 & $8270-13530$ & 136 \\
\hline
\end{tabular}


Tabla 5. Estimaciones poblacionales de las tortugas terrestres gigantes de las islas Galápagos entre 1992 al 2004. $\mathrm{n}=$ número de tortugas capturadas; $\mathrm{N}^{*}=$ población actual estimada; Intervalos = población mínima y máxima estimada; Densidad $/ \mathrm{km}^{2}=$ cantidad de tortugas que actualmente se estima que viven en un kilómetro cuadrado en el sitio de marcación; Fuente $=$ es el autor y año del monitoreo: $\mathrm{CM}=$ Cruz Márquez, $\mathrm{PNG}=$ Parque Nacional Galápagos, Individuos repatriados = Tortugas jóvenes desarrolladas en cautiverio y devueltas a su lugar de origen, como incremento a la población.

\begin{tabular}{|c|c|c|c|c|c|c|c|}
\hline $\begin{array}{l}\text { Isla o } \\
\text { Volcán }\end{array}$ & $\begin{array}{l}\text { Género y } \\
\text { Especie }\end{array}$ & $\mathbf{n}$ & $\mathbf{N}^{*}$ & Intervalo & $\begin{array}{l}\text { Densid. } \\
\mathbf{k m}^{2}\end{array}$ & Fuente & $\begin{array}{l}\text { Indivi. } \\
\text { Repa. }\end{array}$ \\
\hline v. Wolf & G. becki & 426 & 1139 & $276-3568$ & 228 & $\begin{array}{l}\text { CM } \\
1999\end{array}$ & 40 \\
\hline v. Darwin & $\begin{array}{l}\text { G. } \\
\text { microphyes }\end{array}$ & 160 & 818 & $561-1075$ & 205 & $\begin{array}{l}\mathrm{CM} \\
2004\end{array}$ & 0 \\
\hline v. Alcedo & $\begin{array}{l}\text { G. } \\
\text { vandenburghi }\end{array}$ & 2470 & 6320 & $\begin{array}{l}5000- \\
10000\end{array}$ & 799 & $\begin{array}{l}\text { CM } \\
1995\end{array}$ & 0 \\
\hline $\begin{array}{l}\text { v. S. } \\
\text { Negra }\end{array}$ & G. guntheri & 419 & 694 & $400-700$ & 122 & $\begin{array}{l}\mathrm{CM} \\
1995\end{array}$ & 286 \\
\hline v. C. Azul & G. vicina & 671 & 2574 & $\begin{array}{l}1800- \\
2700\end{array}$ & 155 & $\begin{array}{l}\text { CM } \\
1995\end{array}$ & 357 \\
\hline Santa Cruz & G. nigrita & 2310 & 3391 & $\begin{array}{l}2311- \\
6703\end{array}$ & 730 & $\begin{array}{l}\mathrm{CM} \\
2002\end{array}$ & 210 \\
\hline Española & G. hoodensis & 199 & 860 & $800-1200$ & 200 & $\begin{array}{l}\text { CM } \\
2002\end{array}$ & 1293 \\
\hline S.Cristóbal & $\begin{array}{l}\text { G. } \\
\text { chathamensis }\end{array}$ & 1652 & 1824 & $\begin{array}{l}1400- \\
2000\end{array}$ & 559 & $\begin{array}{l}\text { PNG } \\
2004\end{array}$ & 55 \\
\hline Santiago & G. darwini & 366 & 1165 & $891-1370$ & 124 & $\begin{array}{l}\text { CM } \\
2002\end{array}$ & 498 \\
\hline Pinzón & G. ephippium & 235 & 532 & $459-605$ & 134 & $\begin{array}{l}\text { CM } \\
2004\end{array}$ & 552 \\
\hline Pinta & G. abingdoni & 1 & 1 & no aplica & & & \\
\hline Total & & 8909 & 19318 & $\begin{array}{l}13898- \\
29921\end{array}$ & & & 3291 \\
\hline
\end{tabular}

\section{Densidad}

En las zonas de muestreo, las densidades poblacionales fueron calculadas en forma general dentro del área de estudio, sin especificar los lugares donde las tortugas fueron más o menos densas así, para los volcanes Darwin, Sierra Negra, Cerro Azul, Wolf y las islas Pinzón, Española y Santiago, fueron mayores de 100 y 200 tortugas $/ \mathrm{km}^{2}$. Mientras que las de las islas San Cristóbal, Santa Cruz y del volcán Alcedo, las densidades fueron mayores a 500 y 700 tortugas $/ \mathrm{km}^{2}$ respectivamente (Tabla 6 ).

\section{Estructuras Poblacionales:}

En 1992-2002, se observaron diferencias en las estructuras poblacionales entre los machos y las hembras de las tortugas G. vandenburghi, G. nigrita, $G$. vicina $y \quad G$. hoodensis. En $G$. guntheri, $G$. chathamensis y G. darwini; también las hembras y los juveniles, fueron diferentes en la estructura de la población; se exceptúan las poblaciones de $G$. microphyes y $G$. ephippium, que la estructura poblacional en los tres estratos no fue diferente (Tabla 7, Figura 5-b y Figura 6). Estas mismas diferencias fueron observadas en las estructuras poblacionales actuales (1992-2002) comparadas con las del pasado (1964-1974). Las diferencias existentes entre los tres estratos poblacionales $(\hat{\partial},+$ y juveniles $) \mathrm{de}$ las tortugas gigantes que ocurrieron entre las décadas del 60-70 y las actuales 92-02, en los que se observan variaciones, según la isla, volcán o población, (Ji-Cuadrados lo muestran, Tabla 7).

Proporción Sexual

Las marcaciones y recapturas realizadas entre 1992 hasta el 2002, cuatro de las poblaciones, la proporción sexual es próxima a 1:1, se exceptúan $G$. microphyes, guntheri, G. nigrita, G. hoodensis, y $G$. darwini, cuya proporción sexual se encuentra por debajo de $0.8 \bigcirc: 1 \hat{\jmath}$. Pinta no ha podido ser recuperada, a pesar de los esfuerzos desplegados por el PNG y la FCD, y su proporción de sexo se mantiene en $0 \bigcirc: 1 \lesssim$ (Tabla 8). La proporción sexual de las poblaciones de tortugas terrestres gigantes, que determinó MacFarland (1974), no fue $1: 1$, se exceptúa $G$. vandenburghi.

\section{Mortalidad Natural y} Depredación:

El número de tortugas observadas muertas, por causas naturales o sacrificadas por el hombre, en cada una de las poblaciones de tortugas, fue principalmente en Sierra Negra, seguida por la de Cerro Azul, Alcedo y San Cristóbal. En el resto de poblaciones visitadas, solamente se registró restos de individuos por muerte natural, acción que fue más notoria y con mayor abundancia en las poblaciones de Santa Cruz y volcán Alcedo, estos animales no estuvieron destrozados los carapachos por cortes con machete o hacha, y todas las partes corporales estuvieron completas en el lugar (Tabla 9).

\section{Amenazas Potenciales:}

En Cerro Azul, Los Cinco Cerros, se registró el $1.8 \%$ (12/671) con mordeduras antiguas de perros; sobretodo placas caudales anteriores y posteriores destruidas. En esta misma población, se observó el $4.6 \%$ (31/671) de las tortugas más viejas con llagas hechas por las hormigas Solenopsis spp. La Tabla 10, muestra los organismos introducidos y nativos que aun amenazan a las tortugas gigantes en su hábitat natural; 
depredación que ocurre en cualquiera de sus estadios de vida o compiten por el alimento (Figura 6, tortugas destruidas sus placas marginales anteriores $\mathrm{y}$ posteriores por perros cimarrones entre 1980-1983).

Tabla 6. Diferencias estadísticas de las estructuras poblacionales de tortugas terrestres gigantes entre las décadas de 1964 - 1974 con las de $1992-2002 . *=$ Ausencia de información.

\begin{tabular}{|l|l|l|l|l|}
\hline $\begin{array}{l}\text { Isla o } \\
\text { volcán }\end{array}$ & Especie & t & g. l. & Probabilidad \\
\hline v. Wolf & G. becki & $*$ & $*$ & $*$ \\
\hline v. Darwin & G. microphyes & 170.59 & 307 & $<0.0005$ \\
\hline v. Alcedo & $\begin{array}{l}\text { G. } \\
\text { vandenbughi }\end{array}$ & 132.79 & 5607 & $<0.0005$ \\
\hline $\begin{array}{l}\text { v. Sierra } \\
\text { Negra }\end{array}$ & G. guntheri & 29.02 & 771 & $<0.001$ \\
\hline $\begin{array}{l}\text { v. Cerro } \\
\text { Azul }\end{array}$ & G. vicina & 54.73 & 2768 & $<0.0005$ \\
\hline Santa Cruz & G. negrita & $*$ & $*$ & $*$ \\
\hline Española & G. hoodensis & 65.06 & 871 & $<0.0005$ \\
\hline $\begin{array}{l}\text { San } \\
\text { Cristóbal }\end{array}$ & G. & 60.89 & 1889 & $<0.0005$ \\
\hline Santiago & G. darwini & 175.92 & 1550 & $<0.0005$ \\
\hline Pinzón & G. ephippium & 60.23 & 482 & $<0.0005$ \\
\hline Pinta & G. abingdoni & $*$ & $*$ & $*$ \\
\hline
\end{tabular}

\section{Discusión}

\section{Distribución}

En los siglos XVI al XVIII, las poblaciones de tortugas gigantes, fueron abundantes y ampliamente distribuidas en las islas donde habitan (Porter, 1815; Vandenburgh, 1914; Slevin, 1959). La distribución actual, es similar a la distribución que mostró MacFarland et al. (1974), y diferente a la que observaron científicos, coleccionistas, piratas, cazadores de ballenas, focas y los primeros colonos. Según registros de estos hombres, las tortugas se las encontraba por miles y miles en los mismos lugares y poblaciones, donde actualmente son pocos o están extintas (Townsend, 1925). Actualmente, la única población de tortugas estable, que mantiene una amplia distribución y abundancia de individuos, es $G$. vandenburghi de volcán Alcedo. Las poblaciones remanentes de las otras subespecies tienen distribuciones parchadas y aisladas por barreras geográficas de flujos de lava, carreteras, pueblos y otros.

La distribución espacial de las tortugas repatriadas en Española, sexualmente madura, están dispersas en la mayor parte de la isla, siempre prefiriendo los parches de cactos Opuntia megasperma variedad
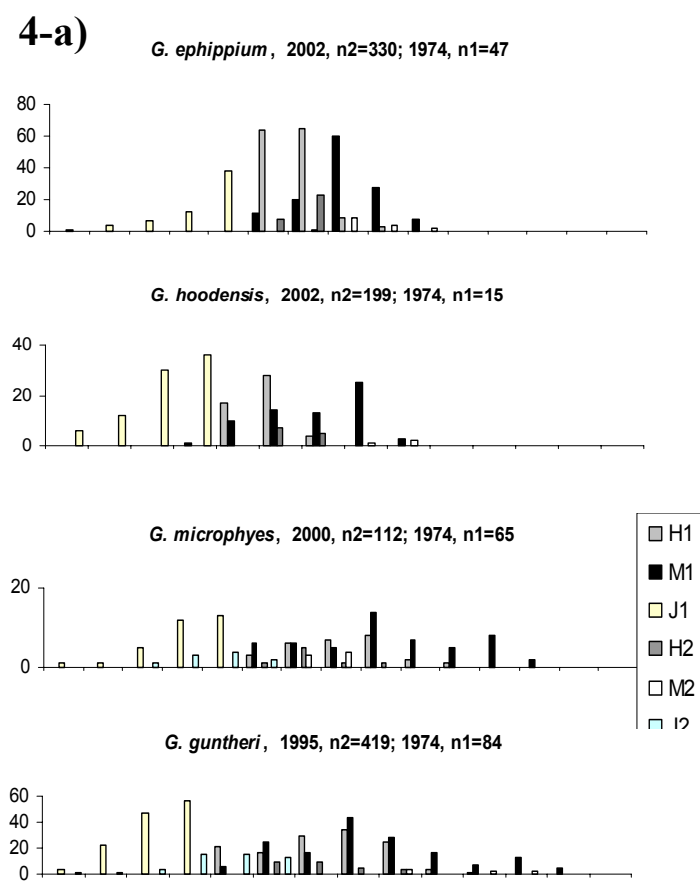

G. darwini, 2002, n2=366; 1974, n1=286

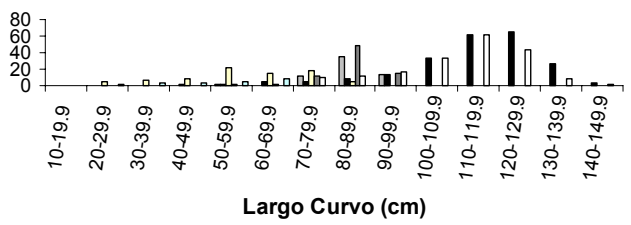

Figura 4a-b. Estructuras poblacionales actuales $\left(\mathrm{M}_{2}\right.$, $\left.\mathrm{H}_{2}, \mathrm{~J}_{2}\right)$ y del pasado $\left(\mathrm{M}_{1}, \mathrm{H}_{1}, \mathrm{~J}_{1}\right)$ de las tortugas terrestres gigantes (Geochelone spp.) de seis islas del Archipiélago de Galápagos. $(\mathrm{a}=$ representa las poblaciones de los volcanes Alcedo, Cerro Azul y Wolf, $G$. vandenburghi, $G$. vicina y $G$. becki respectivamente; San Cristóbal y Santa Cruz; $G$. chathamensis y $G$. nigrita respectivamente, b (en la siguiente Pág.) = muestra las poblaciones de Pinzón, Española y Santiago, G. ephippium, G. hoodensis y $G$. darwini, respectivamente; y los volcanes Darwin y Sierra Negra; G. microphyes y $G$. guntheri, respectivamente.

orientalis en la región central, media y alta de la isla. Con referencia al uso del mismo hábitat, las repatriadas sexualmente maduras, han convergido al mismo ambiente de sus progenitores. Entre 19641974, la distribución de los 14 últimos ejemplares $\left(12\right.$ q y $\left.2{ }^{\top}\right)$ adultos supervivientes, era muy escasa y dispersa, la reproducción ocurría en forma normal, pero el reclutamiento de jóvenes era de cero, la depredación del gavilán de Galápagos en los neonatos y juveniles era el problema (Márquez et al., 1999). Las tortugas preferían vivir bajo los troncos de cactos esperando la caída de una flor, fruto o un cladodio 
para alimentarse, el sobre pastoreo de las cabras había eliminado la mayor parte del forraje en la isla. El mejor método de protección y conservación que optó la $\mathrm{FCD} / \mathrm{PNG}$, fue trasladar a cautiverio los últimos supervivientes de la Española, para reproducirlos y restaurar la población con 1293 individuos a la fecha.

G. $n$. vandenburghi, 1974, n1=291; 1995, n2= 1680
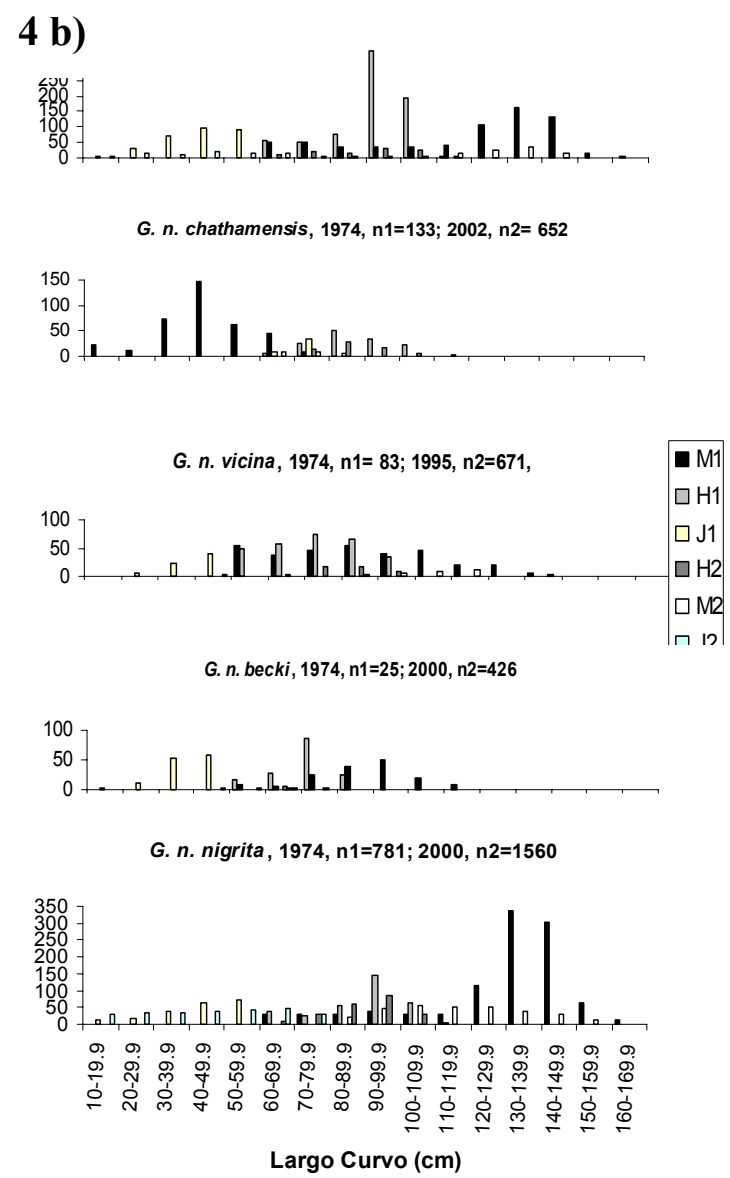

Comportamiento similar es observado en los individuos repatriados sexualmente maduros en las islas Pinzón y Santiago, su distribución geográfica es similar a la de los adultos nativos. Dentro de este mismo contexto, se ha registrado, que las poblaciones de G. vicina, G. guntheri, G. becki y G. chathamensis, que también recibieron en su población jóvenes desarrollados en cautiverio, siguen el mismo patrón ecológico de distribución espacial en el hábitat natural de sus progenitores.

\section{Demografía}

En la actualidad, los tamaños poblacionales de las tortugas gigantes en el Archipiélago, han incrementado o disminuido por diferentes aspectos relacionados con los seres humanos en las islas. Dos ejemplos: $G$. guntheri, continúa disminuyendo su población, por la depredación humana, mientras $G$. hoodensis sigue aumentando por restauración con jóvenes desarrollados en cautiverio a través del trabajo de conservación de la FCD y PNG. Las actividades de conservación incluyen primero, el control $\mathrm{y}$ erradicación de especies introducidas que compiten en el forraje y depredan huevos y neonatos en el hábitat. Segundo, traslado total a cautiverio de los 14 últimos ejemplares supervivientes de la población de la isla Española, para reproducir y criar jóvenes en cautiverio. Tercero, protección de nidos y traslado de huevos y neonatos al Centro de Crianza, y desarrollar crías hasta una edad que puedan sobrevivir en su ambiente. Cuarto, la exploración de nuevos ambientes, que no fueron localizados antes, donde habitan tortugas en la actualidad.

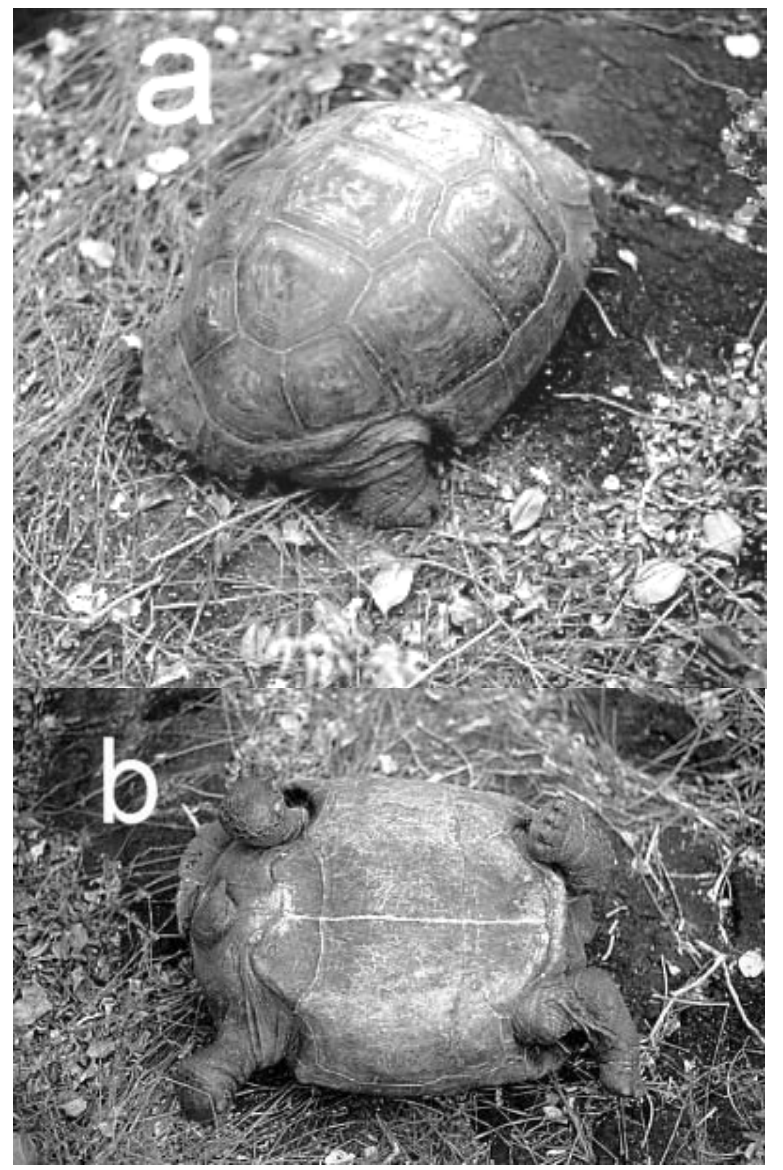

Figura 5a-b. Tortuga gigante subadulta mordida y destruidas algunas de sus placas marginales caudales posteriores derecha, anales y gulares del plastrón por perros cimarrones en la zona de los Cinco Cerros del volcán Cerro Azul en el Sur de la isla Isabela; a = vista dorsal, $\mathrm{b}=$ vista ventral. (Foto: Cruz Márquez, 1994).

Con estas acciones conservacionistas, al menos el $75 \%$ de las especies de tortugas más amenazadas, han superado el cuello de botella en que se encontraron (por ejemplo, G. hoodensis y G. ephippium), a través de la restauración con jóvenes desarrollados en cautiverio. 
La gran amenaza que presentaron algunas poblaciones de tortugas, ameritó que investigadores realicen estudios entre 1962-1964, probando formas de incubar y criar tortugas en condiciones de cautiverio, y los estudios sugirieron establecer un Centro de Crianza en 1965 (Snow, 1964). MacFarland et al. (1974), también reflejaron claramente el problema. Técnicas de mejoramiento de crianza para tortugas, fueron establecidas en 1987 (Snell et al., 1988). Sirvieron para aumentar la población de la isla Española en un 98\%, la de Pinzón el 84\%, Santiago incremente el $56 \%$, Cerro Azul, Sierra Negra y Santa Cruz acrecienten el 54, 45 y $7 \%$ de individuos respectivamente en sus poblaciones con relación a los estimados de 1974. Las poblaciones de tortugas de volcán Wolf y San Cristóbal, recibieron mínimo incremento poblacional, sin embargo $G$ chathamensis, está en mejor estado poblacional, por la ausencia de depredadores. Los 3292 repatriados de todas las especies han fortalecido las distintas poblaciones, posterior a lo estimado por MacFarland (1974).

\section{Densidad}

Las densidades poblacionales estimadas, son menores de 100 tortugas $/ \mathrm{km}^{2}$, las intermedias, menores a 300 tortugas $/ \mathrm{km}^{2}$ y las altas, menores que 800 tortugas $/ \mathrm{km}^{2}$. MacFarland et al. (1974) no determinan estimaciones de densidades poblacionales en ninguna de las poblaciones. No existen estudios científicos previos, que registren densidades de tortugas en el pasado. Observaciones puntuales indican que las tortugas se encontraron en grandes cantidades (por miles), por ejemplo en las pampas de Sierra Negra y otras islas (Darwin, 1835; Rothschild, 1896; Beck, 1903; Townsend, 1925; Slevin, 1931). Densidades actuales aún son menores a 4 tortuga/ha. Densidades mayores a 4 tortuga/ha son localizadas en Alcedo y Santa Cruz (Márquez et al., 1995; Torres, 2000). Según Stoddart \& Savy (1983), las densidades estimadas de Geochelone gigantea en el Atolón de Aldabra, son entre 500 y 7000 tortugas $/ \mathrm{km}^{2}$, equivalente a 5 y 70 tortugas/ha. Las densidades de tortugas en Galápagos se encuentran por debajo y sobre el valor mínimo, con relación a lo estimado en Aldabra. Probablemente en los siglos XVI hasta el XVIII las densidades de las tortugas en el Archipiélago podrían haber sido similares o mayores a las que hoy se registran en el Atolón de Aldabra.

\section{Estructura}

Las estructuras poblacionales de G. microphyes y G. ephippium, no son diferentes en ninguno de los tres estratos poblacionales comparados. Esto indica que a partir de 1974, especialmente G. microphyes, tuvo un bajo incremento poblacional natural, hasta la fecha. Los gavilanes son los limitantes del reclutamiento de jóvenes, haciendo lento el incremento poblacional. En Pinzón con G. ephippium, ratas negras y gavilanes no permiten reclutamiento. La restauración in situ es solo con jóvenes reclutados en cautiverio, e incrementa ciertos tamaños de clase gradualmente y mejora la estructura y tamaño de la población.

La población estructural estratificada de las tortugas $G$. vandenburghi y $G$. nigrita, actual, es sustancialmente diferente con las de 1974, en los tres estratos. Esta morfología estructural poblacional, se relaciona con las tasas de crecimiento de cada uno de los individuos que en el estudio de MacFarland et al. (1974), se asume que fueron animales subadultos y juveniles, actualmente son adultos hembras y machos. Tortugas que nacieron en aquel tiempo, son subadultos y adultos en ciertos casos. También los nacimientos de años posteriores lograron sobrevivir y mantener la población actual, como subadultos y juveniles. Los esfuerzos de muchos años de conservación del SPNG y la FCD particularmente en la población de Santa Cruz, con el control mediante casería de cerdos por parte de guarda parques y la comunidad, han disminuido la depredación de jóvenes y el aumento del reclutamiento en la población.

En las poblaciones de G. hoodensis, G. ephippium, $G$. darwini, $G$. vicina y $G$. guntheri, estructuras poblacionales son reforzadas con jóvenes desarrollados en cautiverio, ganando cierto nivel de restauración, mostrando una imagen diferente de su estructura, con relación a lo que presentaron en 1974. Los estratos juveniles fueron de cero en las islas Española y Pinzón y bajos números en Santiago. También, en Española y Pinzón, los estratos poblacionales de machos y hembras adultas, fueron mínimos. Paralelamente, la erradicación de cerdos cimarrones en Santiago y el control de ratas en Pinzón, son factores que han ayudado un poco en la recuperación de los estratos poblacionales en términos generales.

Estructuras poblacionales de machos y hembras de $G$. vicina y $G$. hoodensis, actuales, no son similares, pero si lo son las de los juveniles con las de 1974. Esto se relaciona con tasas de crecimiento de individuos repatriados (Española). Jóvenes y subadultos registrados en 1974, en la actualidad son adultos, más los individuos repatriados que ahora son adultos (Cerro Azul). La no diferencia en los juveniles, se relaciona: primero, en G. hoodensis, no registró juveniles en 1974, ahora registra el $6.02 \%$ de reclutamiento natural en la población, más los jóvenes repatriados cada año (Márquez et al., 2000; Márquez \& Naranjo, 2002). Segundo, en G. vicina, a pesar de recibir el 54\% de jóvenes repatriados en la población, hasta 1984-1995 que se erradican los perros cimarrones, habían disminuido el $72 \%$ de la restauración total.

La población estratificada actual de G. guntheri, con la de 1974, hembras y juveniles son diferente y no diferente los machos. Esto indica que, hembras, juveniles y subadultos, presentes en 1974 son adultos actualmente, con mayor producción y reclutamiento 
de jóvenes. La población de machos adultos ha recibido poco incremento y mayor depredación humana. La estructura poblacional actual de $G$. chathamensis, con la de 1974, los juveniles son diferente, y no diferente los machos y las hembras. Morfología poblacional que se relaciona con las tasas de sexo; en 1974 la relación de sexo era 0.7우으, actualmente es $19: 1 \delta^{\lambda}$. A mayor cantidad de hembras reproductoras, mayor será la producción de juveniles en la población (Márquez, 1994). De tal manera que la población juvenil actual supera a la de adultos, y también a la de 1974. A esto se suma la erradicación de perros y cerdos cimarrones, favoreciendo el crecimiento poblacional juvenil y de adultos.

La estructura poblacional actual de $G$. darwini, en comparación con la de 1974, los machos son diferente, y no diferente las hembras y juveniles. Esto tiene relación con la incubación de los huevos en condiciones naturales. Las zonas de anidación en Santiago se ubican arriba de los $200 \mathrm{msnm}$, donde las temperaturas de incubación están por debajo de los $28^{\circ} \mathrm{C}$, ocasionando el $75 \%$ de machos en la prole que se desarrolla (Enríquez, 1984; Snell et al., 1988). De tal manera, que las crías y huevos que se trasladan a cautiverio, se encuentran en sus últimos estadios de desarrollo fetal, y los fetos vienen con sexo preestablecido, por tanto, lo que se repatría a la isla, en su mayoría son machos. Permitiendo que la población de hembras se mantenga baja, y la de machos siga en progreso. Por otro lado, en el pasado, la población juvenil se mantuvo limitada por cerdos y gavilanes y a partir del 2000, los cerdos fueron erradicados. Sin embargo los gavilanes aun mantienen limitado el reclutamiento de jóvenes en la isla (Márquez et al., 2002).

\section{Proporción sexual}

La proporción sexual de las poblaciones de tortugas gigantes en la actualidad no es en absoluto similar a lo encontrado en 1974 , en sentido progresivo o negativo, comportamiento ecológico que está relacionado con algunos factores, antropogénicos, ambientales y de conservación a saber: primero, las exploraciones de nuevas áreas habitadas por tortugas permitió localizar y marcar más tortugas en la población. Segundo, el control y erradicación de perros y cerdos en Isabela, Santiago y San Cristóbal, por personal del SPNG y la comunidad, y de las cabras en las islas Española y Pinta, han ayudado en el fortalecimiento de las poblaciones de tortugas. Tercero, los métodos de conservación utilizados desde 1965 hasta 1986 permitieron que poblaciones como la de Española, su proporción de sexo cambiara de $19: 0.3 \hat{\jmath}$ a $0.7 \bigcirc: 1 \hat{\jmath}$, solamente incubando los huevos con incubadora solar, y a partir de 1987, estudios de determinación de sexo por la temperatura en la incubación de los huevos, fue el factor determinante en el aumento de la tasa de machos y hembras en la población de Española (Sancho, 1988; Márquez et al.,
1989). Por otro lado, la intervención humana, el traslado de huevos y neonatos a cautiverio, la crianza de juveniles en cautiverio, permitió restaurar otras poblaciones más amenazadas.

\section{Mortalidad Natural y Depredación}

La depredación en las poblaciones supervivientes de tortugas gigantes de las islas Galápagos es uno de los factores que aún no ha podido ser solucionado. Por un lado, los depredadores cerdos cimarrones recientemente fueron erradicados de la isla Santiago, y hace algunas décadas, fueron eliminados en San Cristóbal. En Isabela y Santa Cruz, personal del SPNG y la comunidad caza a los cerdos para consumo y al mismo tiempo los controla. Las ratas negras en Pinzón y otras islas, donde estas ocurren, son otros depredadores de difícil eliminación. Los perros cimarrones fueron erradicados en Isabela y Santa Cruz, pero aún quedan los domésticos como depredadores potenciales que surgen de vez en cuando en las islas con presencia humana. Los herbívoros competidores y destructores de nidos de tortugas, los burros cimarrones, se mantienen en algunas de las poblaciones de tortugas. El ganado, caballos, y las cabras cimarronas introducidas, continúan en algunas islas.

Las hormigas Solenopsis spp., en las poblaciones de tortugas de Santa Cruz e Isabela Sur se alojan en los nidos, perforan los huevos y se comen embriones y neonatos al eclosionar y atacan la piel de los jóvenes y adultos. La hormiga se transporta a kilómetros de distancia usando la región ventral del carapacho de las tortugas, donde hace también nidos. En todas las poblaciones de tortugas de Isabela, cazadores furtivos sacrifican las tortugas para comerlas, acción que ocurre con más énfasis y mayor abundancia en los volcanes de Cerro Azul y Sierra Negra. En 1994, en San Cristóbal se registraron dos tortugas sacrificadas por cazadores de cabras. En el 2000, Guías del SPNG, en Bahía Urbina, al Oeste del volcán Alcedo, registraron tres carapachos de tortugas adultas, sacrificadas por cazadores. Estas acciones contrastan con los esfuerzos conservacionista que hace más de 40 años la FCD y SPNG vienen desarrollando en pro de la conservación en Galápagos.

\section{Amenazas Potenciales}

Estas acciones indican que una parte de la comunidad galapagueña aún no está conciente que las tortugas son reptiles protegidos; por tanto aún queda mucho trabajo de educación ambiental que hacer, en las islas donde el hombre habita. Dentro de este mismo contexto, el hombre es una amenaza potencial, para las poblaciones de tortugas gigantes en las islas, de igual forma los cerdos cimarrones, los perros domésticos y las hormigas Solenopsis spp. Por lo tanto la protección, para conservar las tortugas gigantes en las islas Galápagos, debe continuar, hasta que las amenazas hayan terminado para siempre. Fritts et al. (2000) sugieren como prioridades principales, 
acciones similares a efectuarse, para poder conservar las especies endémicas de tortugas terrestres gigantes en el Archipiélago.

Para la restauración natural de la población de tortugas G. chathamensis, fue requerida escasa intervención humana. La erradicación de perros, cerdos y el control de los burros, solo fue necesario para que se restaure sin reclutamiento de jóvenes en cautiverio. La población juvenil es ahora superior a la adulta. Modelos como este son requeridos en otras poblaciones, sin presencia de gavilanes, que es el mayor limitante del reclutamiento en las poblaciones de tortugas. Todas las poblaciones de tortugas con presencia de gavilanes requerirán de un mayor reclutamiento de jóvenes en cautiverio, hasta que hayan ganado un alto reclutamiento de hembras, como la de volcán Alcedo. Luego podrán quedar exentas de peligro, por este depredador natural, que por su condición de endémico será permanente. Se asume que las poblaciones de ambos organismos endémicos interactúan, igual como coevolucionaron en el pasado.

\section{Conclusiones}

Al comparar el estado actual (1992 - 2002) de las poblaciones de tortugas terrestres gigantes (Geochelone spp.) en relación a 1974 se determina:

1. La distribución geográfica actual, tiene poca variación con relación a la localizada en 1974.

2. La demografía poblacional actual, varía mucho en sentido progresivo, con relación a los estimados de 1974. Pero se asume que aún las poblaciones son pequeñas, con relación a lo existente entre los siglos XVI-XVIII y los tamaños poblacionales de G. gigantea de Aldabra.

3. Las densidades poblacionales actuales en general, aún son pequeñas, menores a 4 tortuga/ha, con relación a los estimados de Aldabra por Stoddart \& Savy (1983), y mucho menos a lo que pudo haberse estimado en los siglos XVI-XVIII. Actualmente, en el borde Sur del volcán Alcedo, es la única población donde las tortugas están más agrupadas en las épocas frías en Galápagos y las densidades son mayores a 4 tortuga/ha.

4. Con relación a lo mostrado en 1974, las estructuras poblacionales actuales de las tortugas son significativamente diferentes, en algunas al menos son similares y en otras han declinado $G$. guntheri en la actualidad (2004) se consideran menos de 600 individuos en la población.

5. Con relación a lo registrado en 1974, la proporción sexual actual de las tortugas gigantes de Galápagos ha mejorado sustancialmente en un $50 \%$ en ascender a $1: 1$ o al menos próxima a $1: 1$ Comportamiento ecológico relacionado con la intervención humana, en términos de restauración poblacional en algunas de ellas.

6. La depredación de las tortugas en Galápagos, por causas de organismos cimarrones y antropogénicos, es un factor de difícil solución, por las grandes extensiones de áreas silvestres de difícil acceso en los ecosistemas isleños y del tipo de depredador.

7. Las tortugas en general aún mantienen amenazas potenciales múltiples, entre ellas la antropogénica, el gavilán de Galápagos, hormigas de fuego (Solenopsis spp.), ratas negras y los cerdos. La isla Isabela es la más grande del archipiélago donde habitan 5 especies de tortugas gigantes. Las tres especies del Norte se encuentran amenazadas por varios factores; pero aun tienen poblaciones con algunos miles de individuos; mientras en el Sur es otro el problema. Una de las dos especies del Sur $(G$. vicina) aun mantiene una población moderada de miles de individuos. La otra, G. guntheri, sigue en declinación por presión humana, y su población se cree menor a 600 individuos. La FCD/SPNG incrementan investigación, manejo y actividades educativas, en un serio esfuerzo por restaurar la población de $G$. guntheri, la segunda más en peligro de las tortugas de Galápagos.

\section{Agradecimiento}

Nuestra sincera gratitud a los colaboradores, Guarda Parques, Voluntarios y Becarios del $\mathrm{FCD} / \mathrm{PNG}$, que participaron en el campo en los distintos muestreos, para conseguir la información en las poblaciones de tortugas. A las organizaciones internacionales PNUD y UNF, FZS, FOG_SUIZA y otros que a través de la $\mathrm{FCD} / \mathrm{PNG}$, financiaron los distintos proyectos de estudios de tortugas Terrestres Gigantes en Galápagos; ya que, sin su ayuda y apoyo, no hubiera sido posible, terminar este estudio. Al PNG, por permitirnos realizar los estudios pertinentes en las islas, y a la FCD, por la logística y administración de los fondos.

\section{Literatura citada}

Baur G. 1889. The gigantic land tortoises of the Galápagos Islands. Amer. Nat. 23 (276): 10391057.

Beck R.H. 1903. In the home of the giants tortoises. Annual Report of the New York Zoological Society. 7: 160-174.

Caccone A., Gibbs J.P., Ketmaier V., Suatoni E. \& Powell J.R. 1999. Origin and evolutionary relationships of giant Galápagos tortoises. Evolution. 96: 13223-13228.

Caccone A., Gentile G., Gibbs J.P., Fritts T.H., Snell H.L., Betts J. \& Powell J.R. 2002. Phylogeography and History of Giant Galápagos Tortoises. Evolution. 56: 2052-2066.

Ciofi C., Milinkovitch M.C., Gibbs J.P., Caccone A. \& Powell J.R. 2002. Microsatélite análisis of genetic divergence among populations of giant 
galápagos tortoises. Molecular Ecology. 11: $2265-$ 2283.

Crebs C.J. 1989. Ecological Methodology. University of British Columbia. Harper \& Row, Publishers. New York.

Darwin C. 1845. Journal of Research into the Nature History and Geology of the coutries visited during el voyage of the HMS Beagle round the World under the command of Capt. Fitz Roy, R. N. From 1832-1836. 2nd ed. Murria, London.

Ernst C. \& Barbour R. 1989. Turtles of the World. Smithsonian Institution Press.

Enriquez R. 1984. Aspectos Importantes del Ciclo de Vida de la Tortuga de Galápagos Geochelone elephantopus darwini de la Isla Santiago. Tesis de doctorado, Universidad Central del Ecuador, Quito.

Fritts T.H., Snell H.L., Cayot L.J., MacFarland C., Earsom S., Márquez C., Llerena W. \& Llerena F. 2000. Progress and priorities in research for the conservation of reptiles. Bulletin Royal Institut Sciences Naturelles, Belgique. Supplement. 70: $39-45$.

Fritts T.H. 2001. A Brief Review of the Taxonomic History of Galapagos Tortoises Relevant to Consideration of the Most Appropriate Generic and Specific Names for Giant Tortoises in Galapagos. - Draft. In press.

Gunther A. 1875. Description of the living and Extinct Races of Gigantic Land Tortoises. Part I-II, Introduction, and the tortoises of the Galápagos Islands. Philos. Trans. Royal Soc. 165: 251-284.

Gunther A. 1877. Gigantic Land Tortoises Living and Extinct in the Collection of the British Museum. London. 96.

Harland R. 1827. Description of Land tortoise from the Galápagos Islands, Commonly Known as the Elephant Tortoise. J. Acad. Nat. Sci. Philadelphia. 5: 284.

Harland R. 1835. Description of the Testudo elephantopus from the Galápagos Islands. Medical and Physical Researches. 1835: 190-196.

MacFarland C.G., Villa J. \& Toro B. 1974. The Galápagos Giant Tortoises (Geochelone elephantopus). Part I: Status of the surviving Populations. Biological Conservation. 6: 118-133.

Márquez C., Rea S., Snell H.L. \& Llerena F. 1989. Effect of Incubation Temperature on the sex of giants tortoises. In G. Davis-Merlen, ed., 19861987. Ann. Report. CDRS.: 83-84.

Márquez C., Cayot L.J. \& Rea S. 1999. La Crianza de tortugas Gigantes en Cautiverio: un manual operativo. Impreso en: A \& B Editores, Quito, Ecuador.

Márquez C., Snell H.L., Altamirano M., Salazar X. \& Torres M.L. 2000. Ecología de restauración para la diversidad biológica en Galápagos: recuperación de los reptiles endémicos. Informe FUNDACYT.

Porter D. 1815. Journal of a Cruise Made to the Pacific Ocean by Captain David Porter in the United States Frigate Essex in the years 1812, 1813,1814. 2 vols, Philadelphia. Second edition, New York.

Rothschild W. 1896. Further notes on gigantic land tortoises. Novitates Zoologicae. 3(2): 85-99. Sancho A.C. 1988. Influencia de la Temperatura de Incubación en el Sexo y Parámetros para el Reconocimiento del Sexo en la Tortuga Gigante de Galápagos (Geochelone elephantopus) e Histología de la Gónada Iguana Terrestre (Conolophus subcristatus), Tesis de Licenciatura, Pontificia Universidad Católica del Ecuador, Quito.

Slevin J.R. 1931. Log of the Schooner "Academy" on a voyage of Scientific research to the Galapagos Islands. 1905-1906. Ocasional papers of the California Academy of Sciences. 17: 1-162.

Slevin J.R. 1959. The Galapagos Islands: A history of their exploration. Ocasional Papers of the California Academy of Sciences. No. 25.

Snow D.W. 1964. The Giant Tortoises of the Galapagos Islands. Their Present Status and future chances. Oryx. 7: 277-290.

Stoddart D.R. \& Savy S. 1983. Aldabra: island of giant tortoises. Ambio. 12 (3-4): 180-185.

Townsend C.H. 1925. The Galapagos Tortoises in their relation to the whaling industry: a study of old logbooks. Zoológica. 4: 55-135.

VanDenburgh J. 1914. The Gigantic Land Tortoises, of the Galapagos Archipelago.Proc. California Acad. Sci. 2, Part. 1: 203-374.

Zar J.H. 1999. Bioestadistical Análisis. Fourth ed. Prentice-Hall, Englewood Cliffs, N. J. 


\footnotetext{
${ }^{1}$ Estación Científica Charles Darwin, Casilla 17013891 Quito, Isla Santa Cruz, Galápagos-Ecuador Fax: (593-5) 2526146 ó 147

Teléfonos: 2526146 ó 147

Correo electrónico: marquez@ffdarwin.org.ec

${ }^{2}$ Estación Científica Charles Darwin, Casilla 17013891 Quito, Isla Santa Cruz, Galápagos-Ecuador Fax: (593-5) 2526146 ó 147

Teléfonos: 2526146 ó 147

Correo electrónico: dwiedenfeld@fcdarwin.org.ec

${ }^{3}$ Department of Biology, University of New Mexico, Albuquerque, New Mexico, NM87131-USA. Correo electrónico: snell@unm.edu o howard@fcdarwin.org.ec

${ }_{5}^{4}$ Department of Biology, University of New Mexico, Albuquerque, New Mexico, NM87131-USA.

${ }^{5}$ Center for Protected Areas Management and Training

Department NRRT Forestry Building

CSU, Fort Collins, CO 80523-1480. Correo electrónico: craigmacfarland@hotmail.com

${ }^{6}$ Parque Nacional Galápagos, Puerto Ayora, Isla Santa Cruz, Galápagos-Ecuador

Fax: 593-5-526

Teléfono 2526-511 ó 180. Correo electrónico: wtapia@png.org.ec

${ }^{7}$ Parque Nacional Galápagos, Puerto Ayora, Isla Santa Cruz, Galápagos-Ecuador

Fax: 593-5-526

Teléfono 2526-511 ó 180. Correo electrónico: snaranjo@png.org.ec
} 